\title{
Sequential transarterial chemoembolization and early radiofrequency ablation improves clinical outcomes for early-intermediate hepatocellular carcinoma in a 10-year single-center comparative study
}

Liangliang Yan ${ }^{1,2+}$, Yanqiao Ren ${ }^{1,2+}$, Kun Qian ${ }^{1,2}$, Xuefeng Kan ${ }^{1,2}$, Hongsen Zhang ${ }^{1,2}$, Lei Chen ${ }^{1,2}$, Bin Liang ${ }^{1,2}$ and Chuansheng Zheng ${ }^{1,2^{*}}$

\begin{abstract}
Background: Transarterial chemoembolization (TACE) and radiofrequency ablation (RFA) are effective treatment methods for unresectable hepatocellular carcinoma (HCC). However, there is still a lack of clinical research on whether early sequential RFA, compared with late combination therapy, can improve the long-term efficacy of initial TACE treatment.

Methods: This retrospective study investigated a cohort of patients who underwent combination therapy using TACE and RFA (TACE followed by RFA) from January 2010 to January 2020 at our medical centre. A total of 96 patients underwent TACE combined with early RFA (usually during the first hospitalization), which was called TACE + eRFA. Thirty-four patients received 1-2 palliative TACE treatments first and then underwent TACE treatment combined with late RFA (TACE + IRFA). All patients continued to receive palliative TACE treatments after intrahepatic lesion progression until reaching intolerance. The overall survival (OS) rate, time to tumour progression (TTP), tumour response rate and major complication rates were compared between the two groups.

Results: There were significant differences in the median OS (46 months vs 33 months; $P=0.013$ ), median TTP (28 months vs 14 months; $P<0.00)$, objective response rate (ORR) $(89.6 \%$ vs $61.8 \%, P=0.000)$ and disease control rate (DCR) $(94.8 \%$ vs $73.5 \% P=0.002)$ between the two groups. Multivariable analysis revealed that the Barcelona Clinic Liver Cancer stage was an independent risk factor for OS. Meanwhile, multivariable analysis revealed that TACE + eRFA was associated with an enhanced TTP.
\end{abstract}

Conclusion: Early sequential RFA treatment in patients with early-intermediate HCC can improve local tumour control and clinical outcomes while reducing the frequency of TACE treatment. In clinical practice, in HCC patients initially treated with TACE, it is recommended to combine RFA as soon as possible to obtain long-term survival.

*Correspondence: hqzcsxh@sina.com

†Liangliang Yan and Yanqiao Ren equal contributors

1 Department of Radiology, Union Hospital, Tongji Medical College,

Huazhong University of Science and Technology, Wuhan 430022, China

Full list of author information is available at the end of the article

(C) The Author(s) 2021. This article is licensed under a Creative Commons Attribution 4.0 International License, which permits use, sharing, adaptation, distribution and reproduction in any medium or format, as long as you give appropriate credit to the original author(s) and the source, provide a link to the Creative Commons licence, and indicate if changes were made. The images or other third party material in this article are included in the article's Creative Commons licence, unless indicated otherwise in a credit line to the material. If material is not included in the article's Creative Commons licence and your intended use is not permitted by statutory regulation or exceeds the permitted use, you will need to obtain permission directly from the copyright holder. To view a copy of this licence, visit http://creativecommons.org/licenses/by/4.0/. The Creative Commons Public Domain Dedication waiver (http://creativecommons.org/publicdomain/zero/1.0/) applies to the data made available in this article, unless otherwise stated in a credit line to the data. 
Keywords: Transarterial chemoembolization, Early radiofrequency ablation, Hepatocellular carcinoma, Overall survival, Time to tumour progression

\section{Introduction}

Cancer is the first or second cause of premature death (30-69 years old) in 134 countries worldwide [1]. Liver cancer is a common malignancy of the digestive system, and hepatocellular carcinoma (HCC) is the main pathological type of liver cancer and the fourth most common cause of cancer-related death [2]. HCC exhibits an annual prevalence growth trend [3-5]. The international and authoritative Barcelona Clinic Liver Cancer (BCLC) staging system is applied according to the patient's tumour characteristics, liver function and physical status and is widely accepted $[6,7]$. The implementation of surveillance programs for high-risk populations and advances in imaging-based diagnostic technology have increased the rate of early HCC diagnosis [8]. Through novel surgical methods, such as orthotopic liver transplantation or liver resection, early HCC can be cured [9]. Unfortunately, only a portion of $\mathrm{HCC}$ patients are suitable for surgical therapies due to an unfavourable tumour location, the presence of multiple tumours, a poor hepatic reserve and a shortage of donor livers. The 5-year recurrence rate in HCC patients treated with liver resection is as high as $50-70 \%[8-10]$.

Radiofrequency ablation (RFA) is the first-line ablation technique applied through an electrode tip inserted into the target lesion that induces coagulation necrosis and is a widely accepted treatment option for patients with early-stage HCC [11]. Compared with liver resection or liver transplantation, RFA can provide similar survival odds $[9,12]$. However, due to the heat-sink effect that occurs next to large blood vessels, the postoperative recurrence rate is still high, and RFA has a poor curative effect in HCC patients with a high tumour burden [13, 14].

In recent years, transarterial chemoembolization (TACE) has been widely used as a palliative therapy in the treatment of HCC patients who are unsuitable for radical therapies [15]. TACE can cause partial necrosis of tumour cells through a strong cytotoxic effect combined with ischaemia [16]. However, not all feeding arteries can undergo chemoembolization by TACE, since they consist of multiple nodes and can develop de novo collateral arteries $[17,18]$. Local recurrence accounts for the majority of relapses following TACE treatment. However, following repeated TACE sessions, TACE failure/refractoriness often occurs, resulting in decreased overall survival (OS) [19]. Consequently, effective adjuvant therapy is essential to prevent or delay relapses.
Recently, studies have indicated that RFA combined with TACE may improve the local control of tumours with a diameter of over $3 \mathrm{~cm}[20,21]$. However, there have been no reports on the timing of the combination. Consequently, this study analysed whether early sequential RFA treatment, following initial TACE, can improve the long-term efficacy against HCC in such patients compared with late combination treatment.

\section{Methods}

\section{Study design and patient selection}

This was a retrospective study performed in accordance with local and national laws and abiding by the guidelines of the Helsinki Declaration. Approval for this study was obtained from The Ethics Committee of Tongji Medical College, Huazhong University of Science and Technology. The need for informed consent was waived by The Ethics Committee of Tongji Medical College, Huazhong University of Science and Technology.

From January 2010 to January 2020, 436 consecutive patients underwent combination therapy using TACE and RFA (TACE followed by RFA) at our medical centre. Prior to these patients undergoing initial TACE therapy, the treatment strategy was recommended by the multidisciplinary tumour board. Considering the patient's age, any serious comorbidities (such as portal hypertension), the patient's compliance, the tumour location (close to the centre, near large vessels or the diaphragm), the lack of donor livers and the limited efficacy of TACE and RFA alone, some patients were not deemed suitable for liver resection or liver transplantation; these patients were recommended for combination therapy (TACE followed by RFA). The time of RFA following TACE was dependent on the disappearance of symptoms postembolization and the recovery of liver function. In this study, the mean interval between TACE and RFA in the TACE combined with early RFA (TACE + eRFA) group was 6.3 days (1-14 days), and the median interval was 5 days. In the TACE combined with late RFA (TACE + IRFA) group, the mean interval between TACE and RFA was 6.1 days (2-13 days), and the median interval was 5.5 days. There was no significant difference in the interval between the two groups $(P>0.05)$. Prior to undergoing the initial TACE treatment, all patients were informed of the benefits and risks of the combination therapy. TACE + eRFA was defined as early sequential RFA treatment (usually during the first hospitalization) following initial TACE. There were patients who were unwilling to take the risk, 
could not afford the cost of two treatments, or recognized the efficacy of TACE treatment alone but refused to undergo the initial TACE combination therapy. In these patients, following 1-2 palliative TACE treatments, TACE + IRFA was performed on the target lesion.

The diagnosis of HCC depended on the diagnostic criteria of the European Association for the Study of the Liver (EASL) and the American Association for the Study of Liver Disease (AASLD) $[8,10]$. A total of 130 patients met the inclusion criteria and were included in this study. The inclusion criteria consisted of the following: (1) unresectable HCC diagnosed by medical imaging or needle biopsy; (2) Child-Pugh class A or B; (3) number of tumours $\leq 3$; (4) Eastern Cooperative Oncology Group (ECOG) score of 0 ; (5) no evidence of invasion of the portal or hepatic venous branches, extrahepatic metastasis, or uncontrolled ascites; and (6) BCLC stage A/B.
Patients were excluded if the exclusion criteria were met. The exclusion criteria consisted of the following: (1) any previous treatment for HCC; (2) renal failure, cardiac failure or haemorrhagic risk $\left(<50 \times 10^{9} / \mathrm{L}\right)$; (3) other malignancies or liver metastases; and (4) incomplete clinical data (Fig. 1).

\section{TACE procedure}

TACE was performed according to our institutional standard protocol, which has been previously reported [20]. All operators had at least five years of experience in performing TACE procedures. In essence, tumour staining and tumour-feeding arteries were determined by angiography. Consequently, a 2.6-Fr microcatheter (Terumo $^{\mathrm{TM}}$, Japan) was inserted into the tumour-feeding arteries as selectively as possible. First, an emulsion of 2-20 mL of iodized oil (Lipiodol Ultra-Fluid; Laboratoire

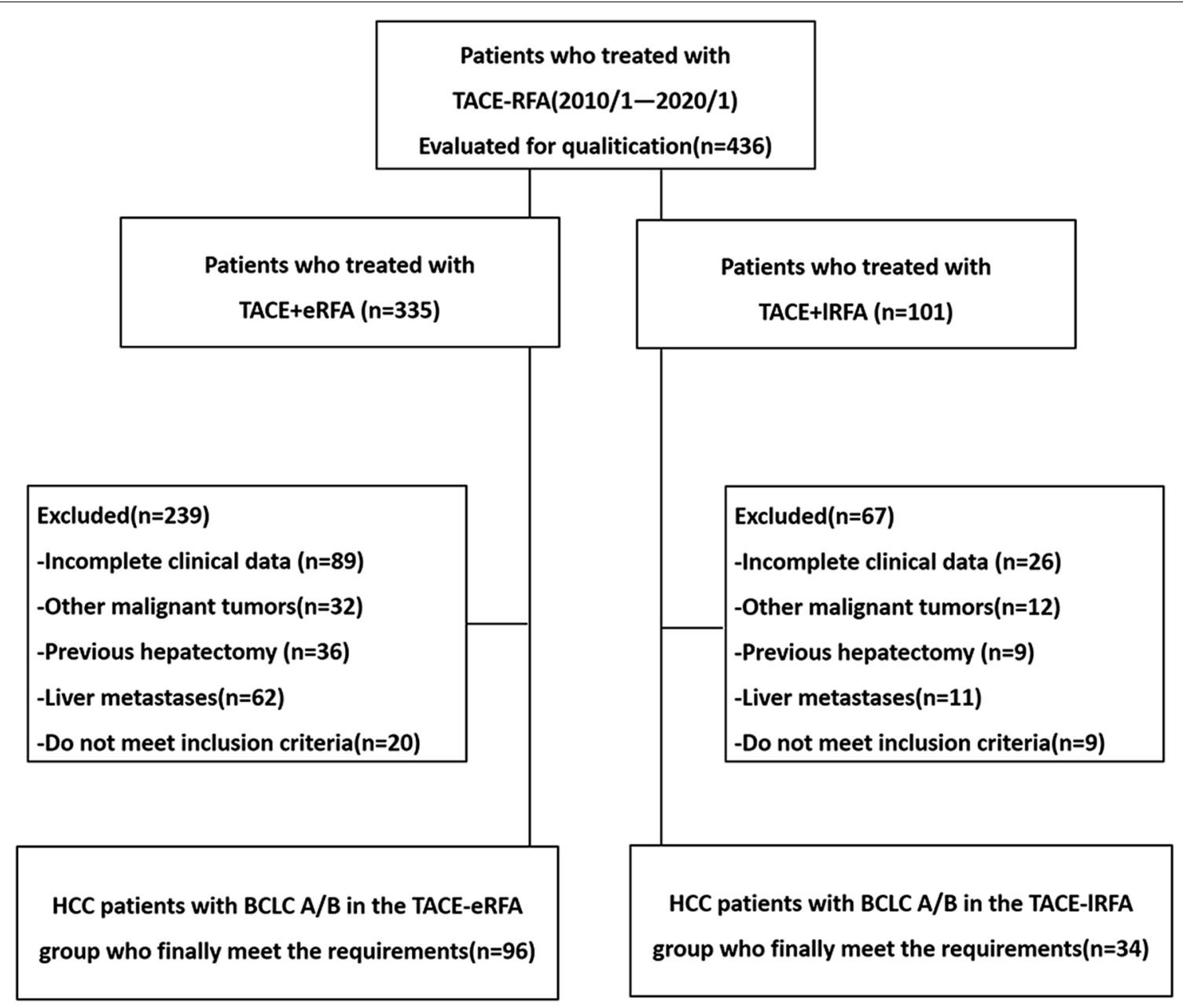

Fig. 1 Enrolment of patients. The flow chart shows the screening procedure for patients with Barcelona Clinic Liver Cancer (BCLC) stage A/B hepatocellular carcinoma (HCC) 
Andre Guerbet, Aulnay-sous-Bois, France) and 20-60 mg of doxorubicin hydrochloride (Hisun Pharmaceutical Co., Ltd., Zhejiang, China) was administered into the target vessels. The dosage of lipiodol and doxorubicin hydrochloride was determined according to the size/vascularity of the tumour and the patient's underlying liver function. Typically, according to the blood supply and tumour diameter, the dosage of iodized oil was 1-3 times the tumour diameter $(1-3 \mathrm{~mL} / \mathrm{cm}$ diameter). For patients with a large tumour burden (usually tumours larger than $10 \mathrm{~cm}$ ) or poor liver function (Child-Pugh score $\geq 8$ ), the initial TACE treatment was performed via fractional embolization or using a reduced amount of emulsion to avoid liver failure. Consequently, gelatine sponge particles (300-500 $\mu \mathrm{m}, \mathrm{Cook}^{\mathrm{TM}}$, Bloomington, Indiana, USA) mixed with contrast material were administered into the tumour-feeding arteries until arterial flow stasis was achieved.

\section{Percutaneous RFA technique}

The RFA procedure was performed in accordance with the standard treatment regimen described in our previous study [20, 22]. In brief, following analgesia (10 mg of morphine) and local anaesthesia (5-10 mL of lidocaine), the electrode needle was inserted into the tumour nodule under the guidance of ultrasound or computed tomography $(\mathrm{CT})$. The RFA procedure was performed with a 460kHz RF generator (Rita Medical Systems, Mountain View, California, USA) and a 14-gauge probe/15-cm-long multiple electrode (Rita Medical Systems, Mountain View, California, USA). To attain a safe range of $0.5-1.0 \mathrm{~cm}$, multiple overlapping ablation zones were required.

\section{Definition and evaluation of data}

The time to tumour progression (TTP) and OS rate were compared between the TACE + eRFA and TACE + IRFA groups. The time of combined treatment to disease progression (local progression or intrahepatic/distant metastasis) was defined as the TTP. OS was defined as the interval between the first TACE procedure and either death or the last follow-up. The modified Response Evaluation Criteria in Solid Tumors (mRECIST) was used to evaluate the treatment response at approximately 1-1.5 months after the combined treatment [23]. A complete response (CR) was defined as the absence of enhancement in all target lesions. A partial response (PR) was defined as at least a $30 \%$ decrease in the sum of the diameters of viable tumours. Progressive disease (PD) was defined as an increase of at least $20 \%$ in the sum of the diameters of the target lesions. Stable disease (SD) was defined as any response that did not qualify as either a PR or PD. Objective tumour regression was defined as a CR or PR. Disease control was defined as a CR, a PR or SD. The Society of Interventional Radiology classification system was also implemented to evaluate the safety of TACE or RFA in both groups [24]. Major complications were defined as events leading to patient death and/ or disability.

\section{Follow-up}

Laboratory tests and contrast-enhanced CT or magnetic resonance imaging (MRI) examinations were performed one month after combined treatment. Imaging (contrastenhanced CT or MRI) and laboratory examinations were performed every 2-3 months for patients. Once local progression or intrahepatic metastasis occurred, palliative TACE treatment was given until it was deemed intolerable by the patient. Follow-up continued until patient death or the end point of this study.

\section{Statistical analyses}

All analyses were performed using SPSS 24.0 software $\left[\mathrm{IBM}^{\mathrm{TM}}\right.$, Armonk, New York], and a $P$ value of $<0.05$ was considered statistically significant. Quantitative data are presented as the mean \pm standard deviation, and discrete variables are presented as proportions. Quantitative data were analysed by Student's t-test, while categorical data were analysed by the chi squared test. The Kaplan-Meier method was used to evaluate differences in the TTP and OS rate between the two groups. Univariate analyses were implemented with the log-rank test. Variables with a $P$ value of $<0.10$ were entered into the multivariate analysis, which was performed using a Cox proportional hazard regression model.

\section{Results}

\section{Study population and patient characteristics}

From January 2010 to January 2020, a total of 436 patients received combination therapy as a treatment option, and 306 patients were excluded since they did not meet the study requirements, as highlighted in Fig. 1. Finally, a total of 130 patients were included in this study, of whom 96 were treated with early sequential RFA treatment after TACE, followed by palliative TACE treatment after subsequent progression. In addition, a total of 36 patients underwent 1-2 TACE procedures, then RFA treatment and finally palliative TACE treatment after tumour progression. The detailed clinical characteristics of the 130 patients are summarized in Table 1 . The median follow-up period was 37 months (range 5.7110.5 months) in the TACE + eRFA group and 33 months (range 14.4-103.9 months) in the TACE+lRFA group. In the TACE + IRFA group, 29 patients died during the observation period, whereas in the TACE + eRFA group, only 58 patients died. 
Table 1 Baseline Characteristics

\begin{tabular}{|c|c|c|c|}
\hline Characteristics & $\begin{array}{l}\text { Early-RFA } \\
(\mathrm{N}=96) \\
(\mathrm{No}, \% ; \text { Mean } \pm \mathrm{SD})\end{array}$ & $\begin{array}{l}\text { Late-RFA } \\
(\mathrm{N}=34) \\
(\mathrm{No}, \% ; \text { Mean } \pm \mathrm{SD})\end{array}$ & $P$ value \\
\hline Gender & & & 0.67 \\
\hline Male & $82(85.4 \%)$ & $28(82.4 \%)$ & \\
\hline Female & $14(14.6 \%)$ & $6(17.6 \%)$ & \\
\hline Age (years) & $55.53 \pm 10.4$ & $54.18 \pm 11.1$ & 0.52 \\
\hline Bilirubin $(\mu \mathrm{mol} / \mathrm{L})$ & $20.3 \pm 13.4$ & $20.1 \pm 11.8$ & 0.94 \\
\hline Albumin (g/L) & $37.8 \pm 6.0$ & $38.0 \pm 5.4$ & 0.87 \\
\hline $\mathrm{PT}(\mathrm{s})$ & $14.3 \pm 1.7$ & $14.0 \pm 1.6$ & 0.31 \\
\hline AST ( $\mu \mathrm{mol} / \mathrm{L})$ & $48.0 \pm 32.4$ & $37.6 \pm 29.9$ & 0.11 \\
\hline Maximal tumor diameter $(\mathrm{cm})$ & & & 0.08 \\
\hline Mean $\pm S D$ & $4.0 \pm 2.8$ & $5.0 \pm 2.7$ & \\
\hline Range & $1.1-15.6$ & $1.9-12.8$ & \\
\hline $1-3 \mathrm{~cm}$ & $44(45.8 \%)$ & $12(35.3 \%)$ & \\
\hline $3.1-5 \mathrm{~cm}$ & $30(31.3)$ & $12(35.3 \%)$ & \\
\hline $5.1-10 \mathrm{~cm}$ & $17(17.7 \%)$ & $8(23.5 \%)$ & \\
\hline$>10 \mathrm{~cm}$ & $5(5.2 \%)$ & $2(5.9 \%)$ & \\
\hline No. of tumors & & & 0.20 \\
\hline Mean \pm SD & $1.18 \pm 0.5$ & $1.29 \pm 0.5$ & \\
\hline 1 & $82(85.4 \%)$ & $24(70.6 \%)$ & \\
\hline $2-3$ & $14(14.6 \%)$ & $10(29.4 \%)$ & \\
\hline Hepatitis & & & 0.56 \\
\hline Hepatitis B & $83(86.5 \%)$ & $28(82.4 \%)$ & \\
\hline Other & $13(13.5 \%)$ & $6(17.6 \%)$ & \\
\hline a-Fetoprotein level & & & 0.95 \\
\hline$>400 \mathrm{ng} / \mathrm{mL}$ & $26(27.1 \%)$ & $9(26.5 \%)$ & \\
\hline$\leq 400 \mathrm{ng} / \mathrm{ml}$ & $70(72.9 \%)$ & $25(73.5 \%)$ & \\
\hline Child-Pugh score & & & 0.67 \\
\hline A & $82(85.4 \%)$ & $28(82.4 \%)$ & \\
\hline B & $14(14.6 \%)$ & $6(17.6 \%)$ & \\
\hline $\mathrm{BCLC}$ & & & 0.17 \\
\hline A & $44(45.8 \%)$ & $11(32.4 \%)$ & \\
\hline B & $52(54.2 \%)$ & $23(67.6 \%)$ & \\
\hline TACE sessions & $3.21 \pm 2.6$ & $5 \pm 2.1$ & 0.00 \\
\hline
\end{tabular}

SD: Standard deviation; PT: Prothrombin time; AST: Aspartate aminotransferase; BCLC: Barcelona Clinic Liver Cancer; TACE: Transcatheter arterial chemoembolization

\section{Treatment response}

The objective tumour regression rate was $89.6 \%$ in the TACE + eRFA group and $61.8 \%$ in the TACE + lRFA group $(P=0.000)$. In addition, the disease control rate was $94.8 \%$ in the TACE + eRFA group and $73.5 \%$ in the TACE + IRFA group $(P=0.002)$. Hence, compared with patients in the TACE + lRFA group, those in the TACE + eRFA group benefitted from an enhanced tumour response.

\section{Complications}

Within the TACE+eRFA group, three patients had severe complications, for an incidence of $2.1 \%$. Two patients developed a biloma following TACE, and one patient suffered from an intestinal perforation after RFA. There were two cases of severe complications in the TACE + IRFA group, for an incidence of $2.9 \%$. A subcapsular haematoma in the liver was observed on CT in two patients. There was no significant difference in the incidence of major complications between the two groups $(P=0.84)$.

OS

The median OS was 46 months (95\%CI: 38.0-54.0) in the TACE + eRFA group and 33 months (95\%CI: 27.438.6) in the TACE + lRFA group $(P=0.013)$ (Fig. 2a). 


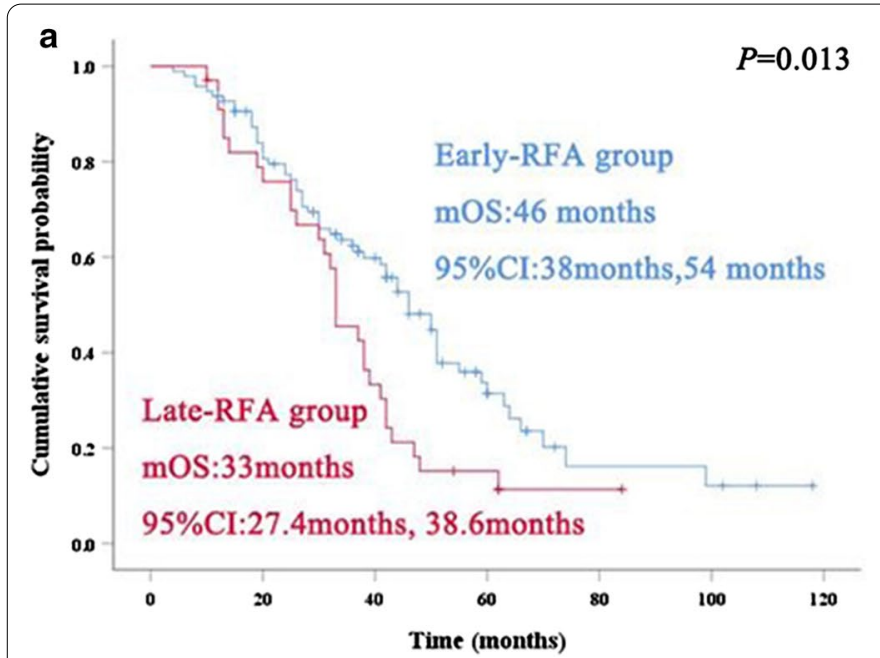

\section{b}

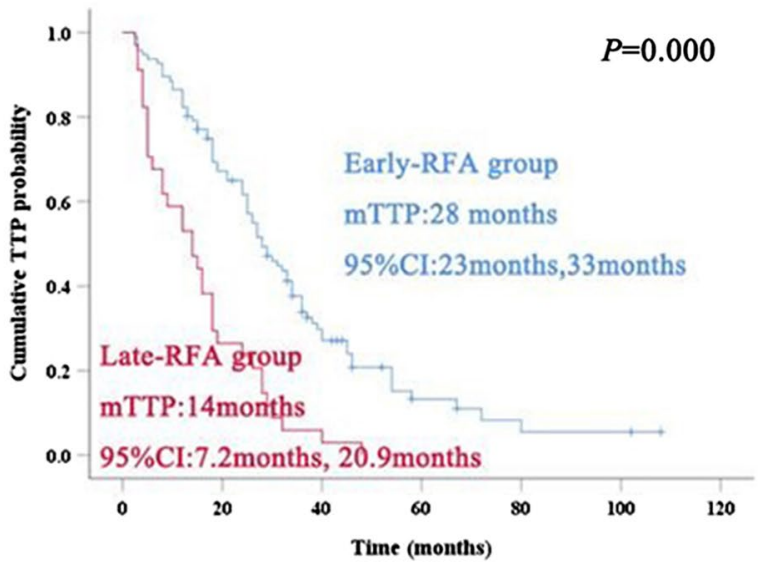

Fig. 2 Kaplan-Meier curves for OS and TTP in the early-RFA (blue line) and late-RFA (red line) groups. a Cumulative survival probability (OS) was significantly longer $(p=0.013$ ) in the early-RFA group (median OS: 46 months, 95\% Cl 38-54) than in the late-RFA group (median OS: 33 months, $95 \% \mathrm{Cl}$ 27.4-38.6). b The cumulative time to tumour progression (TTP) was significantly longer $(p=0.000$ ) in the early RFA group (median TTP: 28 months, 95\% Cl 23-33 months) than in the late RFA group (median TTP: 14 months, 95\% Cl 7.2-20.9 months)

Table 2: Univariate analysis of prognostic factors for overall survival and time to progression

\begin{tabular}{|c|c|c|c|c|}
\hline \multirow[t]{2}{*}{ Variables } & \multicolumn{2}{|l|}{ OS } & \multicolumn{2}{|l|}{ TTP } \\
\hline & $\mathrm{HR}(95 \% \mathrm{Cl})$ & $P$ value & $\mathrm{HR}(95 \% \mathrm{Cl})$ & $P$ value \\
\hline \multicolumn{5}{|l|}{ Gender } \\
\hline Male & 1 & & 1 & \\
\hline Female & $1.213(0.694,2.119)$ & 0.498 & $0.701(0.415,1.183)$ & 0.183 \\
\hline Age (years) & $1.003(0.984,1.023)$ & 0.750 & $1.003(0.986,1.021)$ & 0.715 \\
\hline Bilirubin ( $\mu \mathrm{mol} / \mathrm{L})$ & $1.003(0.986,1.020)$ & 0.759 & $1.004(0.990,1.019)$ & 0.548 \\
\hline Albumin (g/L) & $1.021(0.983,1.060)$ & 0.278 & $0.999(0.967,1.033)$ & 0.972 \\
\hline PT (s) & $0.943(0.816,1.089)$ & 0.423 & $1.006(0.891,1.136)$ & 0.921 \\
\hline AST ( $\mu \mathrm{mol} / \mathrm{L})$ & $1.004(0.997,1.010)$ & 0.294 & $1.005(0.999,1.011)$ & 0.116 \\
\hline Tumor size & $1.179(1.105,1.257)$ & 0.000 & $1.132(1.066,1.201)$ & 0.000 \\
\hline Tumor number & $1.326(0.873,2.015)$ & 0.186 & $1.489(1.032,2.150)$ & 0.033 \\
\hline \multicolumn{5}{|l|}{ Hepatitis } \\
\hline Hepatitis B & 1 & & 1 & \\
\hline Other & $0.861(0.468,1.584)$ & 0.630 & $1.216(0.704,2.100)$ & 0.483 \\
\hline \multicolumn{5}{|l|}{ a-Fetoprotein level } \\
\hline$\geq 400 \mathrm{ng} / \mathrm{mL}$ & 1 & & 1 & \\
\hline$<400 \mathrm{ng} / \mathrm{ml}$ & $1.843(1.169,2.906)$ & 0.008 & $1.738(1.135,2.662)$ & 0.011 \\
\hline \multicolumn{5}{|l|}{ Child-Pugh score } \\
\hline A & 1 & & 1 & \\
\hline B & $0.575(0.337,0.980)$ & 0.042 & $0.614(0.373,1.010)$ & 0.055 \\
\hline \multicolumn{5}{|l|}{ BCLC stage } \\
\hline A & 1 & & & \\
\hline B & $0.348(0.217,0.559)$ & 0.000 & $0.472(0.319,0.700)$ & 0.000 \\
\hline \multicolumn{5}{|l|}{ TACE sessions } \\
\hline 1 & 1 & & 1 & \\
\hline 2 or more & $0.968(0.901,1.040)$ & 0.372 & $1.050(0.991,1.114)$ & 0.101 \\
\hline \multicolumn{5}{|l|}{ Treatment method } \\
\hline Early-RFA & 1 & & & \\
\hline Late-RFA & $0.573(0.365,0.898)$ & 0.015 & $0.351(0.230,0.535)$ & 0.000 \\
\hline
\end{tabular}

OS: Overall survival; TTP: Time to progression; HR: Hazard ratio; CI: Confidence interval; PT: Prothrombin time; AST: Aspartate aminotransferase; BCLC: Barcelona Clinic Liver Cancer; TACE: Transcatheter arterial chemoembolization; RFA: Radiofrequency ablation 
Univariable analyses showed that the mean tumour size, alpha-fetoprotein (AFP) $>400 \mathrm{ng} / \mathrm{mL}$, the Child-Pugh score, the BCLC stage and the therapy method were correlated with OS (Table 2). Consequently, through multivariable analysis (Table 3 ), we identified that the BCLC stage was an independent risk factor for OS $(P=0.038)$.

\section{TTP}

The median TTP was 28 months (95\%CI 28.0-33.0) in the TACE + eRFA group and 14 months (95\% CI 7.2.0$20.9)$ in the TACE + IRFA group $(P<0.001)$ (Fig. 2b). Univariable analyses indicated that the mean tumour size, the tumour number, AFP $>400 \mathrm{ng} / \mathrm{mL}$, the BCLC stage and TACE + eRFA were correlated with the TTP (Table 2). Multivariable analysis revealed that TACE + eRFA was associated with an enhanced TTP (Table 4).

\section{Discussion}

The combination of TACE and RFA has several theoretical advantages over RFA or TACE alone [25, 26]. First, the TACE procedure can reduce the heat-sink effect by inhibiting blood flow in the hepatic artery, thereby expanding the ablation area [27]. In addition, the inclusion of TACE renders the evaluation of ablative margins less challenging and enhances the control of satellite lesions [28]. Conversely, RFA as a radical treatment can lead to a better tumour response, and an enlarged ablation boundary can reduce the formation of a collateral tumour circulation [29-31]. Following repeated TACE procedures, TACE failure/refractory effects often occur [19], leading to a poor tumour response and damaged liver function, eventually causing the upregulation of

Table 3 Multivariate analysis of prognostic factors for overall survival

\begin{tabular}{lll}
\hline Variables & HR $(95 \% \mathrm{Cl})$ & Pvalue \\
\hline $\begin{array}{l}\text { Tumor size } \\
\text { a-Fetoprotein level }\end{array}$ & $1.090(0.991,1.199)$ & 0.075 \\
$>400 \mathrm{ng} / \mathrm{mL}$ & 1 & \\
$\leq 400 \mathrm{ng} / \mathrm{ml}$ & $1.446(0.851,2.456)$ & 0.173 \\
Child-Pugh score & & \\
A & 1 & 0.056 \\
B & $0.587(0.340,1.013)$ & \\
BCLC stage & & \\
A & & \\
B & $0.544(0.306,0.968)$ & \\
Treatment method & & \\
Early-RFA & 1 & 0.038 \\
Late-RFA & $0.659(0.415,1.049)$ &
\end{tabular}

HR: Hazard ratio; Cl: Confidence interval; BCLC: Barcelona Clinic Liver Cancer; RFA: Radiofrequency ablation
Table 4 Multivariate analysis of prognostic factors for time to progression

\begin{tabular}{lll}
\hline Variables & $\mathrm{HR}(95 \% \mathrm{Cl})$ & $P$ value \\
\hline Tumor size & $1.036(0.939,1.143)$ & 0.480 \\
Tumor number & $1.464(0.962,2.230)$ & 0.075 \\
a-Fetoprotein level & & \\
$>400 \mathrm{ng} / \mathrm{mL}$ & $1.580(0.949,2.631)$ & 0.079 \\
$\leq 400 \mathrm{ng} / \mathrm{ml}$ & & \\
Child-Pugh score & 1 & 0.074 \\
A & $0.623(0.370,1.048)$ & \\
B & \\
BCLC stage & $0.662(0.398,1.102)$ & 0.113 \\
A & 1 & \\
B & $0.397(0.252,0.625)$ & 0.000 \\
Treatment method & \\
Early-RFA &
\end{tabular}

HR: Hazard ratio; Cl: Confidence interval; BCLC: Barcelona Clinic Liver Cancer; RFA: Radiofrequency ablation

vascular endothelial growth factor (VEGF) and hypoxia inducible factor (HIF) expression and ultimately reducing the OS rate. Early sequential RFA treatment can cause complete tumour necrosis and inhibit the upregulation of VEGF and HIF expression after hypoxia due to TACE, which reduces local progression and improves patient survival [32].

Presently, there is a lack of relevant literature on the timing of combination therapy. Most studies recommend RFA treatment within two weeks post-TACE [23-27] to better restore liver function. According to one school of thought, a time interval of 0-2 days is sufficient to allow for liver functional reserve recovery in patients with cirrhosis [25]. However, others believe that a shorter time interval deteriorates patient liver function. A period of 3-5 weeks is the optimal time interval for suitable patients to undergo combination therapy, with a well-balanced clinical prognosis [33].

In this study, due to various reasons, some patients failed to undergo combination therapy within two weeks; they underwent 1-2 TACE procedures before treatment and continued to receive palliative TACE treatments after progression. Our results demonstrate that early sequential RFA treatment (Fig. 3) provides better local control, reduced the number of TACE procedures, and prolongs both TTP and survival compared with late combined treatment (Fig. 4). Consequently, if RFA is available, it should actively be recommended to HCC patients as soon as possible. This study indicates that early combined therapy can improve the therapeutic efficacy in patients with HCC. 


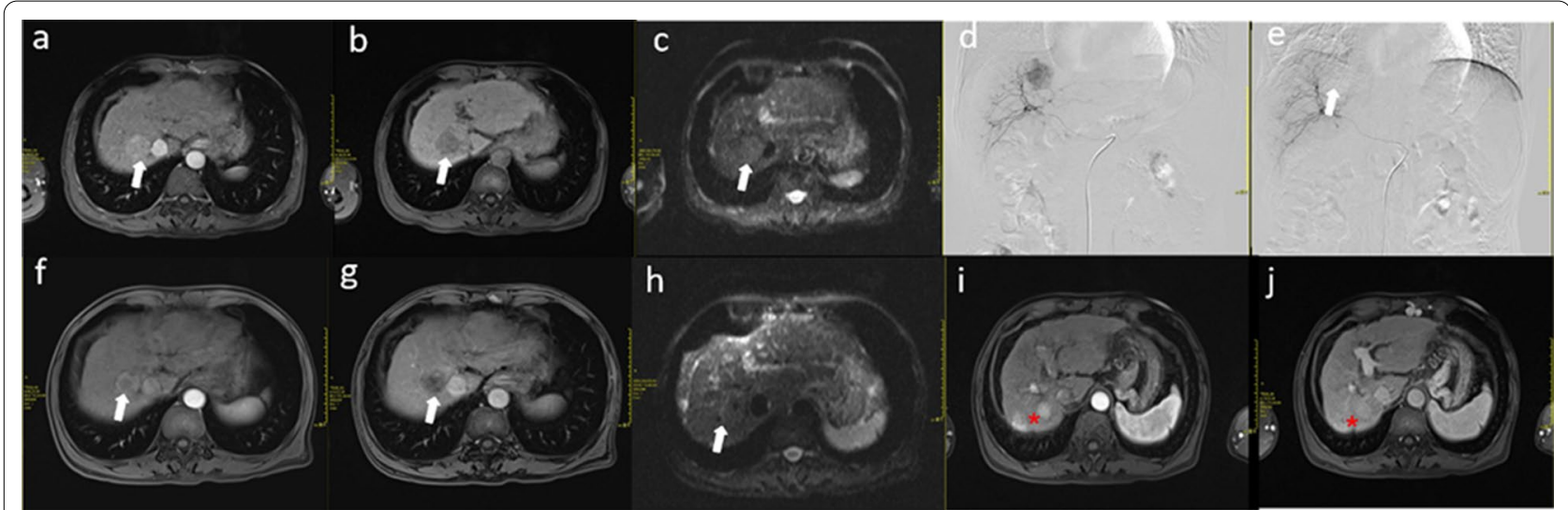

Fig. $3 \mathrm{MRI}$ and digital subtraction angiography (DSA) images of a patient receiving early RFA (5 days) after TACE treatment. a-c The lesion (white arrow) showed a rapid increase in signal intensity during the hepatic arterial phase and the portal venous phase; the entire lesion (white arrow) showed rapid washout of the contrast material and became hypointense compared with the surrounding liver parenchyma. Diffusion-weighted imaging (DWI) showed the dispersion constant. $\mathbf{d}$, e DSA images before and after the initial TACE treatment; the tumour staining disappeared (white arrow). $\mathbf{f}-\mathbf{h}$ One month after combined treatment, the entire lesion (white arrow) showed necrotic foci in the centre, and DWI showed no dispersion constant. $\mathbf{i}$-j After 48 months, there were two lesions in the liver, indicating long-term recurrence (red star sign)

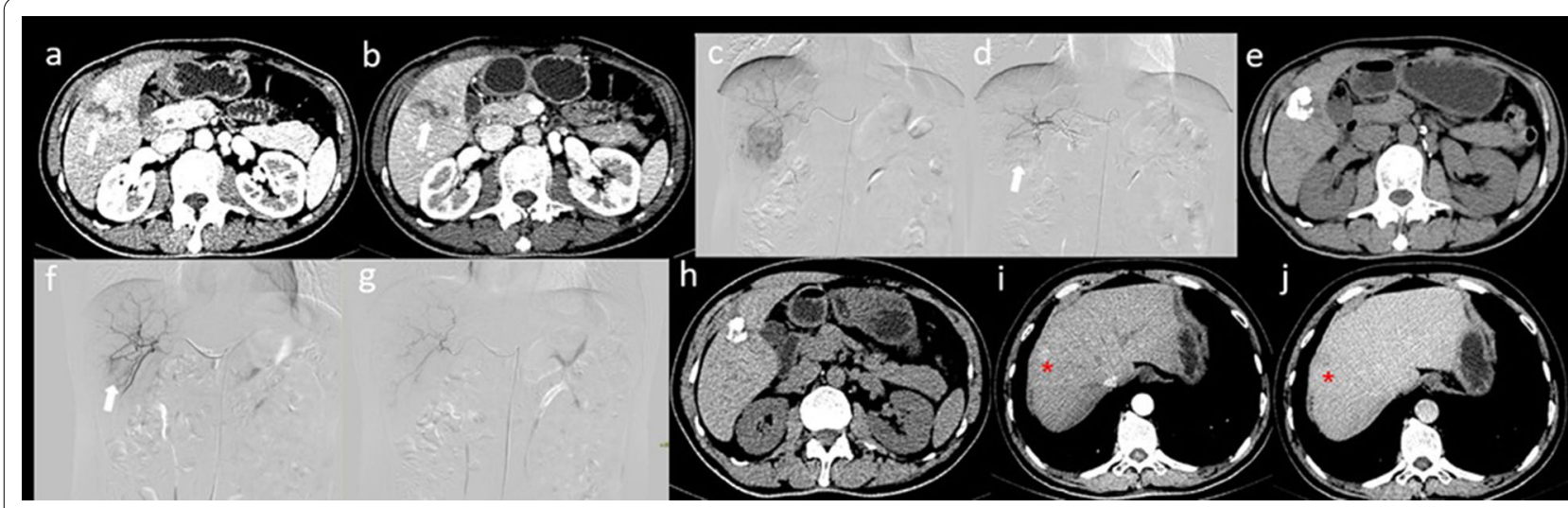

Fig. 4 CT and DSA images of a patient receiving 1 TACE treatment before combination therapy. $\mathbf{a}$, b Axial CT showed a typical HCC enhancement pattern, including "wash in" in the arterial phase, "wash out" in the portal phase (white arrow) in the marginal zone, and necrotic foci in the centre. c, $\mathbf{d}$ DSA images before and after the initial TACE treatment; the tumour staining disappeared (white arrow). e Re-examination after one and a half months showed that the tumour had decreased in size and that viable tumours were present on the edges. $\mathbf{f}, \mathbf{g}$ During the second TACE treatment, the tumour still showed staining (white arrow), indicating that the tumour blood vessels were recanalized and that the tumour was alive. The staining disappeared after treatment. $\mathbf{h}$ Re-examination after the combination therapy showed that the tumour had decreased in size and that there were no live foci. $\mathbf{i}, \mathbf{j}$ Twelve months after late combination therapy, there was recurrence in the liver (red star sign)

Similar to other studies [12, 32, 34], our study revealed that the tumour size, AFP level, Child-Pugh score, and BCLC stage are prognostic factors for OS and TTP. Notably, early sequential RFA treatment was an independent prognostic factor for TTP and is generally safe; there was no significant difference in the incidence of major complications between the two groups.

However, the sample size was small, and the retrospective, non-randomized experimental design was the main flaw of the study. Consequently, a prospective, randomized controlled trial is necessary to verify our results. In addition, due to the limited number of samples, no stratified analysis was conducted.

\section{Conclusions}

In essence, early sequential RFA treatment in patients with early- and mid-stage HCC can improve local tumour control and improve clinical outcomes, such as OS and TTP, while reducing the frequency of TACE treatment. In clinical practice, for HCC patients initially treated with TACE, it is recommended to combine RFA as soon as 


\section{possible to improve the long-term survival of individual HCC patients.}

\begin{abstract}
Abbreviations
BCLC: Barcelona Clinic Liver Cancer; RFA: Radiofrequency ablation; eRFA: Early radiofrequency ablation; IRFA: Late radiofrequency ablation; mRECIST: The modified Response Evaluation Criteria in Solid Tumors; CR: Complete response; PR: Partial response; SD: Stable disease; PD: Progressive disease: VEGF: Vascular endothelial growth factor; HIF: Hypoxia inducible factor; AFP: Alpha-fetoprotein; CT: Computed tomography; EASL: European Association for the Study of the Liver; AASLD: American Association for the Study of Liver Disease: Hepatocellular carcinoma; MR: Magnetic resonance; OS: Overall survival; TTP: Time to tumour progression; TACE: Transarterial chemoembolization.
\end{abstract}

\section{Acknowledgements}

This work was supported by grant from National Nature Science Foundation of China (81873919 and 81701800).

\section{Authors' contributions}

LLY, YQR, QK, XFK, BL, and HSZ collected the patients' data. LLY drafted the manuscript. LLY, YQR and CSZ revised the manuscript. QK, XFK and LC analyzed and interpreted the data. CSZ made substantial contributions to the conception of the work. LLY, YQR and CSZ made substantial contributions to the design of the work, and have revised the manuscript substantively. All authors read and approved the final manuscript.

\section{Funding}

This work was supported by grant from National Nature Science Foundation of China (81873919 and 81701800).

\section{Availability of data and materials}

The datasets used and/or analyzed during the current study are available from the corresponding author on reasonable request.

\section{Declarations}

\section{Consent to publish}

Not applicable.

\section{Ethics approval and consent to participate}

This was a retrospective study and performed in accordance with local and natinal laws and the guidelines of the Helsinki Declaration. Approval for this study was obtained from The Ethics Committee of Tongji Medical College, Huazhong University of Science and Technology. The need for informed consent was waived by The Ethics Committee of Tongji Medical College, Huazhong University of Science and Technology.

\section{Competing interests}

All authors declare that they have no conflict of interest.

\section{Author details}

1 Department of Radiology, Union Hospital, Tongji Medical College, Huazhong University of Science and Technology, Wuhan 430022, China. ${ }^{2}$ Hubei Key Laboratory of Molecular Imaging, Wuhan 430022, China.

Received: 28 November 2020 Accepted: 12 April 2021 Published online: 20 April 2021

\section{References}

1. Sung H, Ferlay J, Siegel RL, Laversanne M, Soerjomataram I, Jemal A, Bray F: Global cancer statistics 2020: GLOBOCAN estimates of incidence and mortality worldwide for 36 cancers in 185 countries. CA Cancer J Clin 2021.

2. Bray F, Ferlay J, Soerjomataram I, Siegel RL, Torre LA, Jemal A. Global cancer statistics 2018: GLOBOCAN estimates of incidence and mortality worldwide for 36 cancers in 185 countries. CA Cancer J Clin. 2018;68(6):394-424.

3. Siegel RL, Miller KD, Jemal A. Cancer statistics, 2020. CA Cancer J Clin. 2020;70(1):7-30.

4. Zheng RS, Sun KX, Zhang SW, Zeng HM, Zou XN, Chen R, Gu XY, Wei WW, He J. Report of cancer epidemiology in China, 2015. Zhonghua Zhong Liu Za Zhi. 2019:41(1):19-28.

5. Zhou M, Wang H, Zeng X, Yin P, Zhu J, Chen W, Li X, Wang L, Wang L, Liu $Y$, et al. Mortality, morbidity, and risk factors in China and its provinces, 1990-2017: a systematic analysis for the Global Burden of Disease Study 2017. Lancet. 2019:394(10204):1145-58.

6. Forner A, Reig M, Bruix J. Hepatocellular carcinoma. Lancet. 2018;391(10127):1301-14.

7. Villanueva A. Hepatocellular Carcinoma. N Engl J Med. 2019;380(15):1450-62.

8. Heimbach JK, Kulik LM, Finn RS, Sirlin CB, Abecassis MM, Roberts LR, Zhu AX, Murad MH, Marrero JA. AASLD guidelines for the treatment of hepatocellular carcinoma. Hepatology. 2018;67(1):358-80.

9. Liu PH, Hsu CY, Hsia CY, Lee YH, Huang YH, Chiou YY, Lin HC, Huo TI. Surgical resection versus radiofrequency ablation for single hepatocellular carcinoma $\leq 2 \mathrm{~cm}$ in a Propensity Score Model. Ann Surg. 2016;263(3):538-45.

10. European Association for the Study of the Liver. Electronic address eee, European Association for the Study of the L: EASL Clinical Practice Guidelines: Management of hepatocellular carcinoma. J Hepatol. 2018;69(1):182-236.

11. Nault JC, Sutter O, Nahon P, Ganne-Carrie N, Seror O. Percutaneous treatment of hepatocellular carcinoma: State of the art and innovations. J Hepatol. 2018:68(4):783-97.

12. Kim YS, Lim HK, Rhim H, Lee MW, Choi D, Lee WJ, Paik SW, Koh KC, Lee $\mathrm{JH}$, Choi MS, et al. Ten-year outcomes of percutaneous radiofrequency ablation as first-line therapy of early hepatocellular carcinoma: analysis of prognostic factors. J Hepatol. 2013;58(1):89-97.

13. Chen J, Peng K, Hu D, Shen J, Zhou Z, Xu L, Chen J, Pan Y, Wang J, Zhang $Y$, et al. Tumor location influences oncologic outcomes of hepatocellular carcinoma patients undergoing radiofrequency ablation. Cancers (Basel). 2018;10(10):378.

14. Kang TW, Lim HK, Lee MW, Kim YS, Rhim H, Lee WJ, Gwak GY, Paik YH, Lim HY, Kim MJ. Aggressive intrasegmental recurrence of hepatocellular carcinoma after radiofrequency ablation: risk factors and clinical significance. Radiology. 2015;276(1):274-85.

15. Lencioni R, de Baere T, Soulen MC, Rilling WS, Geschwind JF. Lipiodol transarterial chemoembolization for hepatocellular carcinoma: a systematic review of efficacy and safety data. Hepatology. 2016;64(1):106-16.

16. Lencioni R. Loco-regional treatment of hepatocellular carcinoma. Hepatology. 2010;52(2):762-73.

17. Takayasu K. Transarterial chemoembolization for hepatocellular carcinoma over three decades: current progress and perspective. Jpn J Clin Oncol. 2012:42(4):247-55.

18. Sakr OS, Berndt S, Carpentier G, Cuendet M, Jordan O, Borchard G. Arming embolic beads with anti-VEGF antibodies and controlling their release using LbL technology. J Control Release. 2016;224:199-207.

19. Kudo M, Matsui O, Izumi N, lijima H, Kadoya M, Imai Y, Okusaka T, Miyayama S, Tsuchiya K, Ueshima K, et al. JSH consensus-based clinical practice guidelines for the management of hepatocellular carcinoma: 2014 update by the Liver Cancer Study Group of Japan. Liver Cancer. 2014;3(3-4):458-68.

20. Ren Y, Cao Y, Ma H, Kan X, Zhou C, Liu J, Shi Q, Feng G, Xiong B, Zheng C. Improved clinical outcome using transarterial chemoembolization combined with radiofrequency ablation for patients in Barcelona clinic liver cancer stage A or B hepatocellular carcinoma regardless of tumor size: results of a single-center retrospective case control study. BMC Cancer. 2019;19(1):983.

21. Peng ZW, Zhang YJ, Liang HH, Lin XJ, Guo RP, Chen MS. Recurrent hepatocellular carcinoma treated with sequential transcatheter arterial chemoembolization and RF ablation versus RF ablation alone: a prospective randomized trial. Radiology. 2012;262(2):689-700.

22. Kan X, Wang Y, Han P, Yao Q, Qian K, Xiong B, Zheng C. Combined ultrasound/computed tomography guidance in percutaneous radiofrequency ablation after transarterial chemoembolization for hepatocellular carcinoma in the hepatic dome. Cancer Manag Res. 2019;11:7751-7. 
23. Lencioni R, Llovet JM. Modified RECIST (mRECIST) assessment for hepatocellular carcinoma. Semin Liver Dis. 2010;30(1):52-60.

24. Ahmed M, Solbiati L, Brace CL, Breen DJ, Callstrom MR, Charboneau JW, Chen MH, Choi Bl, de Baere T, Dodd GD 3rd, et al. Image-guided tumor ablation: standardization of terminology and reporting criteria-a 10-year update. Radiology. 2014;273(1):241-60.

25. Carmi L, Georgiades C. Combination percutaneous and intraarterial therapy for the treatment of hepatocellular carcinoma: a review. Semin Intervent Radiol. 2010;27(3):296-301.

26. Li W, Ni CF. Current status of the combination therapy of transarterial chemoembolization and local ablation for hepatocellular carcinoma. Abdom Radiol (NY). 2019:44(6):2268-75.

27. Rossi S, Garbagnati F, Lencioni R, Allgaier HP, Marchiano A, Fornari F, Quaretti P, Tolla GD, Ambrosi C, Mazzaferro V, et al. Percutaneous radiofrequency thermal ablation of nonresectable hepatocellular carcinoma after occlusion of tumor blood supply. Radiology. 2000;217(1):119-26.

28. Kang SG, Yoon CJ, Jeong SH, Kim JW, Lee SH, Lee KH, Kim YH. Singlesession combined therapy with chemoembolization and radiofrequency ablation in hepatocellular carcinoma less than or equal to $5 \mathrm{~cm}$ : a preliminary study. J Vasc Interv Radiol. 2009:20(12):1570-7.

29. Kim JW, Kim JH, Won HJ, Shin YM, Yoon HK, Sung KB, Kim PN. Hepatocellular carcinomas $2-3 \mathrm{~cm}$ in diameter: transarterial chemoembolization plus radiofrequency ablation vs. radiofrequency ablation alone. Eur J Radiol. 2012;81(3):189-93.
30. Choe WH, Kim YJ, Park HS, Park SW, Kim JH, Kwon SY Short-term interval combined chemoembolization and radiofrequency ablation for hepatocellular carcinoma. World J Gastroenterol. 2014;20(35):12588-94.

31. Haochen W, Jian W, Li S, Tianshi L, Xiaoqiang T, Yinghua Z. Transarterial chemoembolization plus multi-imaging-guided radiofrequency ablation for elimination of hepatocellular carcinoma nodules measuring 3.1 to 5.0 cm: a single-center study. J Int Med Res. 2018;46(7):2650-7.

32. Ni JY, Liu SS, Xu LF, Sun HL, Chen YT. Meta-analysis of radiofrequency ablation in combination with transarterial chemoembolization for hepatocellular carcinoma. World J Gastroenterol. 2013;19(24):3872-82.

33. Feng $Y M$, Wang $X$, Wang L, Ma XW, Wu H, Bu HR, Xie XY, Qi JN, Zhu Q. Efficacy and safety of combination therapy of chemoembolization and radiofrequency ablation with different time intervals for hepatocellular carcinoma patients. Surg Oncol. 2017;26(3):236-41.

34. Peng ZW, Zhang YJ, Chen MS, Xu L, Liang HH, Lin XJ, Guo RP, Zhang YQ, Lau WY. Radiofrequency ablation with or without transcatheter arterial chemoembolization in the treatment of hepatocellular carcinoma: a prospective randomized trial. J Clin Oncol. 2013;31(4):426-32.

\section{Publisher's Note}

Springer Nature remains neutral with regard to jurisdictional claims in published maps and institutional affiliations.
Ready to submit your research? Choose BMC and benefit from:

- fast, convenient online submission

- thorough peer review by experienced researchers in your field

- rapid publication on acceptance

- support for research data, including large and complex data types

- gold Open Access which fosters wider collaboration and increased citations

- maximum visibility for your research: over $100 \mathrm{M}$ website views per year

At BMC, research is always in progress.

Learn more biomedcentral.com/submissions 